\title{
A new system for regulated functional gene expression for gene therapy applications: Nuclear delivery of a p16INK4A-estrogen receptor carboxy terminal fusion protein only in the presence of estrogen
}

\author{
TOMOHIRO TAMURA, TATSUYA KANUMA, TOMOKO NAKAZATO, \\ LERI S. FARIED, HIROSHI AOKI and TAKASHI MINEGISHI
}

\begin{abstract}
Department of Gynecology and Reproductive Medicine, Gunma University Graduate School of Medicine 3-39-22 Showamachi, Maebashi, Gunma 371-8511, Japan
\end{abstract}

Received November 11, 2009; Accepted January 8, 2010

DOI: 10.3892/ijo_00000569

\begin{abstract}
The clinical use of gene therapy requires tight regulation of the gene of interest and functional expression only when it is needed. Thus, it is necessary to develop ways of regulating functional gene expression with exogenous stimuli. Many regulatable systems are currently under development. For example, the tetracycline-dependent transcriptional switch has been successfully employed for in vivo preclinical applications. However, there are no examples of regulatable systems that have been employed in human clinical trials. In the present study, we established an adenovirus-delivered functional gene expression system that is regulated by estrogen. This system uses p16INK4A fused at its $\mathrm{C}$-terminus to the ligand-binding domain of the estrogen receptor $(\triangle \mathrm{ER} \alpha)$. We were able to establish cell lines expressing this gene wherein the functional expression of p16INK4A is estrogen-dependent and causes the arrest of several ovarian cancer cell lines. This inducible and adenovirus-mediated gene transfer system may allow gene therapy using nuclear functioning genes in postmenopausal or ovariectomized women.
\end{abstract}

\section{Introduction}

The success and safety of gene therapy for clinical applications depend on whether functional gene expression can be turned on only at the time of need. Most of the systems reported for the regulation of gene are based on the transactivation of promoter genes by antibiotics, steroid hormones and

Correspondence to: Dr Tatsuya Kanuma, Department of Gynecology and Reproductive Medicine, Gunma University Graduate School of Medicine, 3-39-22 Showamachi, Maebashi, Gunma 371-8511, Japan

E-mail: tkanuma@gunma-cc.jp

Key words: p16INK4A, human estrogen receptor $\alpha$, ovarian cancer, gene therapy, cell cycle tissue-specific cues, for example, tetracycline and mifepristone. It is necessary that the system for regulating functional gene expression can be rapidly and efficiently switched on or off. Such a switch is critical not only when the therapy is no longer needed but also in the case of the development of adverse side-effects to the therapy.

Many regulatable gene expression systems are currently under development. Of these, the tetracycline-dependent transcriptional switch has been used successfully used in vivo for preclinical applications. The tetracycline system is the most widely exploited amongst the existing systems for regulating inducible gene transcription because it has many advantages (1). For example, tetracycline-based systems can facilitate the regulation of targeted gene expression through the use of cell type-specific promoters (2). There are several other drug- or hormone-regulated systems, such as rapamycinand progesterone analogue-inducible systems $(3,4)$, and transactivation systems using tetracycline or fusions of the C-terminal domain of the estrogen receptor (ER) have been reported (5). These systems are useful for controlling target gene expression via the transactivation of steroid hormone receptors.

There are some challenges that remain for regulatable gene expression systems, including leaky gene expression in the off-state. It is essential that this is addressed, especially for proteins that can be toxic when expressed over a long period of time. Stringent regulation of therapeutic gene expression within specific cells or in a localized anatomical region by means of cell type-specific and/or targeted vector systems coupled to efficient transcriptional switches will significantly decrease the risks of negative side-effects, but the complete suppression of expression is not dependent on the gene transcription regulatory systems.

In this study, we established a system for expressing the p16INK4A protein so that it is delivered to the nucleus only in the presence of estrogen. This system uses a p16INK4A protein fused at its $\mathrm{C}$-terminus to the ligand-binding domain of the $\operatorname{ER} \alpha(\Delta \mathrm{ER} \alpha)$, which mediates estrogen-dependent nuclear import. The vector encoding the expression of this protein is delivered by an adenovirus. We show that it is possible to establish cell lines expressing the p16INK4A- 
$\Delta \mathrm{ER} \alpha$ fusion protein and that p16INK4A function in these cells depends on the presence of estrogen.

The p16INK4A protein is a strong inhibitor of the cell cycle at $\mathrm{G} 1$, wherein retinoblastoma $(\mathrm{Rb})$ proteins are in an intact state. Several reports have indicated that p16INK4A is one of the key molecules for replacement gene therapy in cancer cells that show aberrant cell proliferation due to a loss of p16INK4A (6-9). We were able to cause cell cycle arrest in several ovarian cancer cell lines by infection with the adenovirus followed by treatment with estrogen. Therefore, the inducible and adenovirus-mediated gene transfer system can be used in clinical trials of gene compensatory therapy for some nuclear functioning genes in postmenopausal or ovariectomized women, because if the toxic gene overexpression would be observed in the normal cells, the function of gene will not express in the estrogen-free conditions.

\section{Materials and methods}

Cell lines. Ten human ovarian cancer cell lines were used in the current study, including RMG-1 and RTSG (generous gift of Dr Shiro Nozawa, Keio University, Japan); ES-2, TOV21G, OV-90, SKOV3, CaOV3 and PA-1 (American Type Culture Collection, Manassas, VA, USA); and KURAMOCHI and MCAS (Health Science Research Resources Bank, Tokyo, Japan). All cell lines were grown in suitable medium containing $10 \%$ fetal bovine serum (FBS) (Table I).

Reverse transcription (RT)-PCR and Western blot analysis. Total RNA was extracted from cell lines using an RNA isolation system (Promega, Madison, WI, USA). RT-PCR was performed using an RNA PCR Kit (Takara Bio Inc., Shiga, Japan) according to the manufacturer's instructions. The primer sequences for p16INK4A were 5'-CACCATGG AGCCGGCGGCGGGGAGC-3' (sense) and 5'-TCTCGAG ATCGGGGATGTCTGAGGGACC-3' (antisense).

Cells were lysed and analysed by SDS-PAGE and Western blotting using antibodies to one of the following proteins: p16INK4A (Santa Cruz Biotechnology, Inc., Santa Cruz, CA, USA), Rb (Abcam, Inc., Cambridge, MA, USA), cyclindependent kinase 4 (CDK4; Exalpha Biologicals, Inc., Watertown, MA, USA), ER $\alpha$ (Santa Cruz Biotechnology), or anti-ß-actin (Sigma, St. Louis, MO, USA), and detected chemiluminescence.

Plasmid construction. The primer sequences for generating blunt-ended p16INK4A cDNA were 5'-CACCATGGAGCC GGCGGCGGGGAGC-3' (sense primer containing the CACC sequence for directional cloning) and 5'-TCTCGAGATCGG GGATGTCTGAGGGACC-3' (antisense primer containing an XhoI site). The pSG5-ER $\alpha$ plasmid (for expressing the full-length cDNA of the human ER $\alpha$ ) was kindly provided by Dr Pierre Chambon (Institut de Genetique et de Biologie Moleculaire et Cellulaire, CNRS/INSERM/Universite Louis Pasteur/College de France). This plasmid was used as a template for generating a deletion mutant of ER $\alpha$ lacking the N-terminal DNA-binding domain $(\Delta \mathrm{ER} \alpha)$. To prepare this construct, the plasmid was amplified with the following primers: 5'-GTGAACTCGAGTCTGCTGGAGACATGAG AGCT-3' (sense) and 5'-TTAGTCTCGAGTCAGACTGTGG
CAGGGAAACC-3' (antisense). Both primers contained an XhoI restriction site. Both amplified fragments were digested with XhoI and ligated into the pENTR Directional TOPO vector (Invitrogen, Carlsbad, CA, USA).

Adenovirus production. The ViraPower adenoviral expression system (Invitrogen) was used to generate adenovirus vectors. As described by the manufacturer's protocol, LR recombination was performed between $\mathrm{pENTR/D-TOPO-}$ $\mathrm{p} 16 \mathrm{INK} 4 \mathrm{~A}-\Delta \mathrm{ER} \alpha$ and $\mathrm{pAd} / \mathrm{CMV} / \mathrm{V} 5-\mathrm{DEST}$ (Invitrogen). The recombinant adenovirus vector was digested by $P a c \mathrm{I}$ and was transfected into 293A cells using Lipofectamine 2000 (Invitrogen). Two weeks later, cell and culture media were harvested and then subjected to three cycles of freezing and thawing. Crude viral lysate (first seed) was used to infect 293A cells, and the second seed was obtained after approximately 2 weeks. The viral titer was determined as the $50 \%$ tissue culture infectious dose in 293A cell monolayers.

Adenovirus infection and protein expression. All cell lines were routinely maintained in medium containing phenol red. Prior to infection, cells were incubated in phenol red-free Dulbecco's modified essential medium (DMEM) containing $10 \%$ charcoal-treated FBS. For all subsequent experiments, the cells were then infected with adenoviral vectors at a multiplicity of infection of 50-100. After $24 \mathrm{~h}$, the medium was replaced with fresh medium containing $1 \mu \mathrm{M} 173$-estradiol. After an additional $48 \mathrm{~h}$, cells were lysed, and expression of the $\mathrm{p} 16 \mathrm{INK} 4 \mathrm{~A}-\Delta \mathrm{ER} \alpha$ fusion protein was assessed by Western blotting using antibodies against p16INK4A (Santa Cruz Biotechnology), ER $\alpha$ (Santa Cruz Biotechnology), and B-actin (Sigma). Four experimental groups were analyzed: group A, infected cells treated with 17ß-estradiol; group B, infected cells without $17 ß$-estradiol treatment; group $\mathrm{C}$, uninfected cells treated with 173 -estradiol; and group D, cells neither infected nor treated with 17ß-estradiol.

Immunohistochemical analysis. The 10 different types of ovarian cancer cells were plated in phenol red-free DMEM containing $10 \%$ charcoal-treated FBS, and four experimental groups (A-D) were tested. Cells in groups A and B were infected $24 \mathrm{~h}$ after plating, while groups $\mathrm{C}$ and D were not infected. In addition, groups $\mathrm{A}$ and $\mathrm{C}$ were treated with $1 \mu \mathrm{M}$ $17 ß$-estradiol $24 \mathrm{~h}$ after infection. Immunohistochemical analysis was performed $48 \mathrm{~h}$ after the addition of 17ß-estradiol using an antibody against p16INK4A to detect the subcellular localization of $\mathrm{p} 16 \mathrm{INK} 4 \mathrm{~A}-\Delta \mathrm{ER} \alpha$ fusion protein. Monolayer cells were fixed with $95 \%$ ethanol. Non-specific staining was eliminated by incubating with $10 \%$ normal goat serum. The specimens were then reacted for $1 \mathrm{~h}$ with 1:200 rabbit polyclonal antibody against p16INK4A (Santa Cruz Biotechnology). Immunoreactive protein was then detected using a Histofine SAB-PO ${ }^{\circledR}$ kit (Nichirei, Tokyo, Japan) according to the manufacturer's instructions, followed by colorimetric staining with diaminobenzadine (DAB), and the sections were counter-stained with hematoxylin.

Assay of growth suppression. The 10 different ovarian cancer cell lines were plated in phenol red-free DMEM containing $10 \%$ charcoal-treated FBS, and four experimental groups 
Table I. Western blot analyses of ovarian cancer cell lines.

\begin{tabular}{|c|c|c|c|c|c|c|}
\hline \multirow[b]{2}{*}{ Cell line } & \multirow[b]{2}{*}{ Origin } & \multirow{2}{*}{$\frac{\text { RT-PCR }}{\mathrm{p} 16}$} & \multicolumn{4}{|c|}{ Western blotting } \\
\hline & & & p16 & $\mathrm{Rb}$ & $\mathrm{Cdk} 4$ & $\mathrm{ER} \alpha$ \\
\hline SKOV3 & Serous $^{\mathrm{a}}$ & - & - & + & + & + \\
\hline RMG-1 & Clear cell ${ }^{\mathrm{b}}$ & - & - & + & + & + \\
\hline RTSG & Serous ${ }^{\mathrm{a}}$ & - & - & + & + & + \\
\hline MCAS & Mucinous $^{\mathrm{a}}$ & + & - & + & + & + \\
\hline OV90 & Serous ${ }^{\mathrm{a}}$ & + & + & - & + & + \\
\hline $\mathrm{CaOV} 3$ & Serous $^{\mathrm{a}}$ & + & + & - & + & + \\
\hline TOV21G & Clear cell ${ }^{\mathrm{b}}$ & + & + & + & ++ & + \\
\hline $\mathrm{ES}-2$ & Clear cell ${ }^{\mathrm{b}}$ & + & + & + & ++ & + \\
\hline KURAMOCHI & Undifferentiated ${ }^{\mathrm{b}}$ & + & + & + & ++ & + \\
\hline PA-1 & Teratocarcinoma & + & + & + & ++ & + \\
\hline
\end{tabular}

${ }^{\mathrm{a} C y s t a d e n o c a r c i n o m a, ~}{ }^{\mathrm{b}}$ Adenocarcinoma.

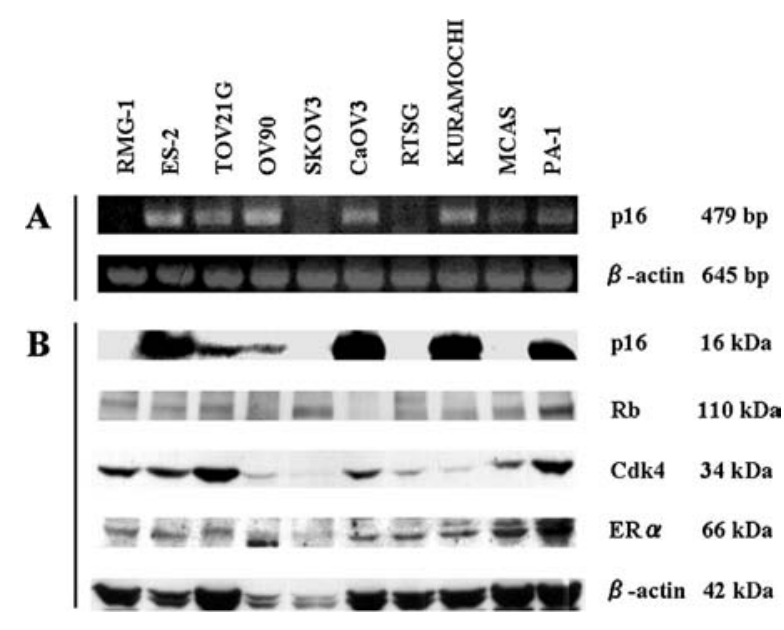

Figure 1. Expression of p16INK4A in ovarian cancer cells. (A) RT-PCR analysis of the p16INK4A gene in ovarian cancer cell lines. $B$-actin cDNA (645 bp) was amplified as a quantitative control. Amplification of p16INK4A cDNA (479 bp) was not detected in RMG-1, RTSG and SKOV3 cells. (B) Western blots for p16INK4A, Rb, CDK4, ER $\alpha$ and $\beta$-actin (control) in ovarian cancer cell lines. Detectable levels of the p16INK4A protein were detected in all cell lines except RMG-1, RTSG, SKOV3 and MCAS. In contrast, $\mathrm{Rb}$ was not detected in OV90 and $\mathrm{CaOv} 3$ cell lines but was detected in the remaining eight cell lines. Detectable levels of the CDK4 and $\mathrm{ER} \alpha$ proteins were found in all 10 cell lines.

(A-D) were tested on the same condition of immunohistochemistry. Cell growth was assessed by MTS assay (Promega). For measurements, $20 \mu \mathrm{l}$ of MTS was added, and the absorbance at $490 \mathrm{~nm}$ was measured after $3 \mathrm{~h}$ using a microplate reader. In one experiment, cell growth was assessed only $48 \mathrm{~h}$ after the addition of $17 ß$-estradiol, and in a second experiment, cell growth was assessed $0,12,24$ and $48 \mathrm{~h}$ after the addition of 17ß-estradiol. Each experiment was repeated 10 times, and the average absorbance at $490 \mathrm{~nm}$ was calculated. $P$ values were calculated using Student's t-test.

Western blot analysis of phosphorylated-Rb protein. To determine whether the transported $\mathrm{p} 16 \mathrm{INK} 4 \mathrm{~A}$ protein inhibits the function of CDK4, cells in groups A-D were analyzed for a decrease in $\mathrm{Rb}$ phosphorylation by Western blotting using an anti-phospho-Rb (Ser780) antibody (Medical \& Biological Laboratories Co., Nagoya, Japan).

Cell cycle analysis using flow cytometry. Forty-eight hours after the addition of 173 -estradiol, $1 \times 10^{6}$ cells in groups A-D were trypsinized and washed with cold phosphate-buffered saline (PBS) and fixed with ice-cold $70 \%$ ethanol. After ethanol was removed, the cell pellets were resuspended in $500 \mu \mathrm{l}$ of PBS, treated with $50 \mu \mathrm{g} / \mathrm{ml}$ of RNase A, and stained with $50 \mu \mathrm{g} / \mathrm{ml}$ of propidium iodide (PI) at $37^{\circ} \mathrm{C}$ for 30 min (10). The DNA content was analysed using the FACScan Coulter Epics XL flow cytometer (Beckman Coulter, Fullerton, CA). The distribution of cells in the different phases of the cell cycle was analysed from the DNA-histograms using EXPO 32 software.

\section{Results}

RT-PCR and Western blot analysis in 10 ovarian cancer cell lines. We examined the expression of p16INK4A mRNA in 10 ovarian cancer cell lines by RT-PCR. Three of the cell lines (RMG-1, RTSG and SKOV3) had no detectable expression of p16INK4A mRNA (Fig. 1A, Table I). Thus, these three cell lines were thought to have a homozygous deletion of the p16INK4A gene.

Next, we examined the levels of p16INK4A, Rb, CDK4, $\mathrm{ER} \alpha$ and $\beta$-actin proteins in these cell lines by Western blotting (Fig. 1B, Table I). We were unable to detect p16INK4A protein in four of the cell lines (RMG-1, RTSG, SKOV3 and MCAS). This is probably due to homozygous deletion or missense mutations in both p16INK4A alleles or to transcriptional silencing. For example, although we detected the mRNA for p16INK4A in MCAS cells, we were unable to detect the p16INK4A protein, a finding consistent with previous reports that these cells have a missense mutation in both alleles of this gene $(11,12)$. These four cell lines all expressed detectable levels of the $\mathrm{Rb}$ protein. In contrast, in 
A.
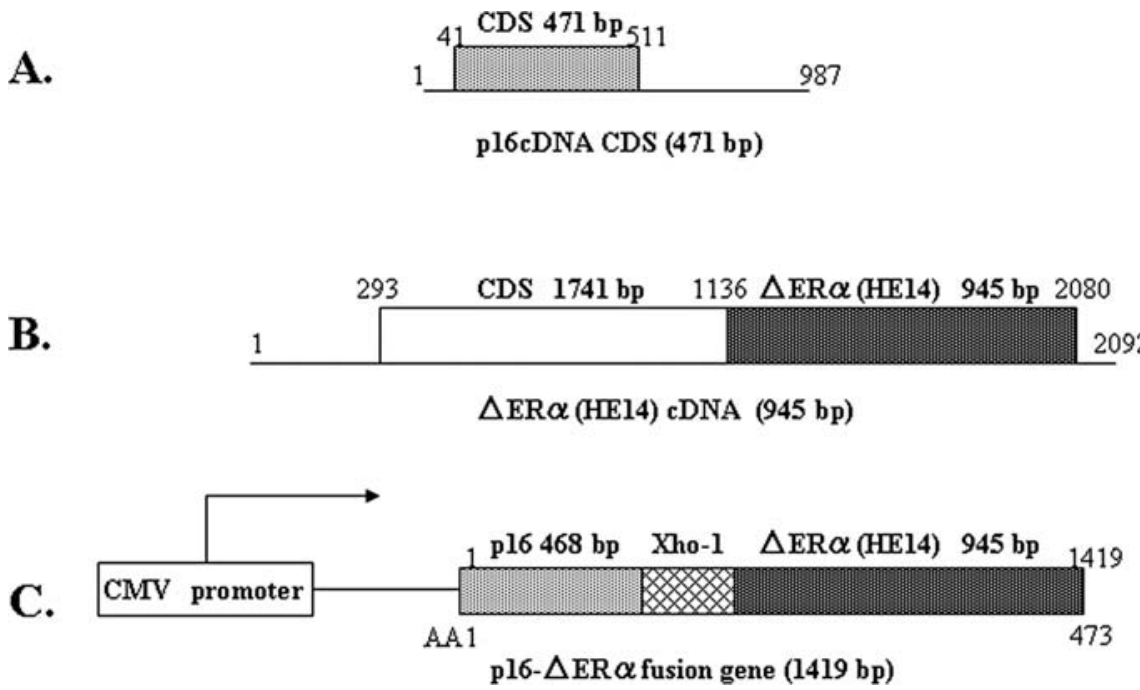

Figure 2. Schematic illustrations of the p16INK4A- $\Delta \mathrm{ER} \alpha$ fusion gene. (A) The p16INK4A cDNA coding sequence (471 bp; nucleotides 41-511). (B) The $\Delta \mathrm{ER} \alpha$ (HE14) cDNA sequence (945 bp; nucleotides 1136-2080; coding sequence nucleotides 293-2080) (13,14). (C) The p16INK4A- $\Delta$ ER $\alpha$ fusion gene was generated by removing the stop codon (TGA) from the p16INK4A cDNA coding sequence and linking it to the $\Delta \mathrm{ER} \alpha$ (HE14) cDNA sequence via the XhoI restriction sites.

OV90 and CaOv3 cells, p16INK4A, but not Rb was detected. These results agree with a previous report that cancer cell lines expressing $\mathrm{Rb}$ fail to express p16INK4A protein and, conversely, that cells expressing p16INK4A rarely have detectable levels of $\mathrm{Rb}$ protein (11). The remaining four cell lines (TOV21G, ES-2, KURAMOCHI and PA-1) expressed both p16 INK4A and Rb proteins. However, whether CDK4 gene amplification (an alternative mechanism for preventing $\mathrm{Rb}$-mediated growth regulation) occurs in these four cell lines remains to be determined. Finally, detectable levels of the ER $\alpha$ protein were found in all 10 cell lines.

Construction of the p16INK4A- $\triangle E R \alpha$ fusion gene and adenovirus production. The recombinant DNA construct $\mathrm{pENTR/}$ D-TOPO-p16INK4A- $\Delta \mathrm{ER} \alpha$ was designed to express the fusion protein as a soluble cytosolic protein following adenoviral infection. In the presence of an ER ligand, the p16INK4A protein is transported into the nucleus, and p16INK4A should carry out its normal function as a specific inhibitor of CDK4. The carboxy terminus of p16INK4A (Fig. 2A) was fused to the ligand-binding domain of $\mathrm{ER} \alpha$, which we refer to as $\triangle E R \alpha$ (product of the HE14 gene with a deletion of the region encoding the DNA-binding domain; Fig. 2B) $(13,14)$. Restriction length polymorphism analysis confirmed the correct orientation of the insert, and sequencing analysis confirmed that there were no frame shifts. The final fusion gene, pAd/CMV/V5-DEST-p16INK4A- $\Delta$ ER $\alpha$ (Fig. 2C), was generated by reaction with LR Clonase. Correct recombination was confirmed by restriction enzyme analysis using XhoI. This adenovirus vector was used to transfect 293 A cells.

Confirmation of infection with the adenovirus and expression of the fusion protein. The p16INK4A- $\Delta \mathrm{ER} \alpha$ fusion protein was detected at the same position of Western blots using antibodies to both p16INK4A and ER $\alpha$ in all virus-infected cell lines (groups A and B) (Fig. 3). No bands were observed in uninfected cells (groups $\mathrm{C}$ and $\mathrm{D}$ ). These results confirm the production of the protein following infection and indicated that the immunological epitope of p16INK4A was conserved despite being attached to the ligand-binding domain of ER $\alpha$.

Immunohistochemical analysis of the subcellular expression of p16INK4A and the p16INK4A- $\Delta E R \alpha$ fusion protein. We performed immunohistochemical analyses to examine the intracellular localization of the fusion protein in estrogenpositive or -negative conditions (Fig. 4) to confirm that the $\mathrm{p} 16 \mathrm{INK} 4 \mathrm{~A}-\Delta \mathrm{ER} \alpha$ fusion protein is expressed in the cytosol after infection but quickly translocated into the nucleus upon addition of estrogen. In these experiments, we used an antibody to p16INK4A to visualize both the p16INK4A$\triangle \mathrm{ER} \alpha$ fusion protein and the wild-type p16INK4A protein. Positive staining was observed in all four cell lines that lacked wild-type p16INK4A (RMG-1, RTSG, SKOV3 and MCAS) when they were infected with the adenovirus (groups A and B). Positive staining was not observed when these cells were left uninfected (groups $\mathrm{C}$ and $\mathrm{D}$ ). When the infected cells were treated 17ß-estradiol, p16INK4A was concentrated in the nucleus (group A), and when 17ß-estradiol was absent, p16INK4A was detected only in the cytosol (group B).

This phenomenon was also observed in all six cell lines expressing wild-type p16INK4A (ES-2, TOV21G, OV90, CaOV3, KURAMOCHI and PA-1). Specifically, staining for p16INK4A was observed in the nuclei and/or cytosol in all six cell lines, but it was stronger in groups A and B (with infection) than in groups $\mathrm{C}$ and $\mathrm{D}$ (without infection). In uninfected cells, only nuclear staining for p16INK4A staining was observed. In contrast, p16INK4A was concentrated in the nucleus of infected cells when they were treated with 17ß-estradiol (group A), but when 17ß-estradiol was absent (group B), p16INK4A was concentrated in the cytosol. Furthermore, the strongest nuclear staining was observed in group A (infected and treated with $17 ß$-estradiol). These results suggest that the $\mathrm{p} 16 \mathrm{INK} 4 \mathrm{~A}-\Delta \mathrm{ER} \alpha$ fusion protein migrated into the nucleus in the presence of an estrogenic 


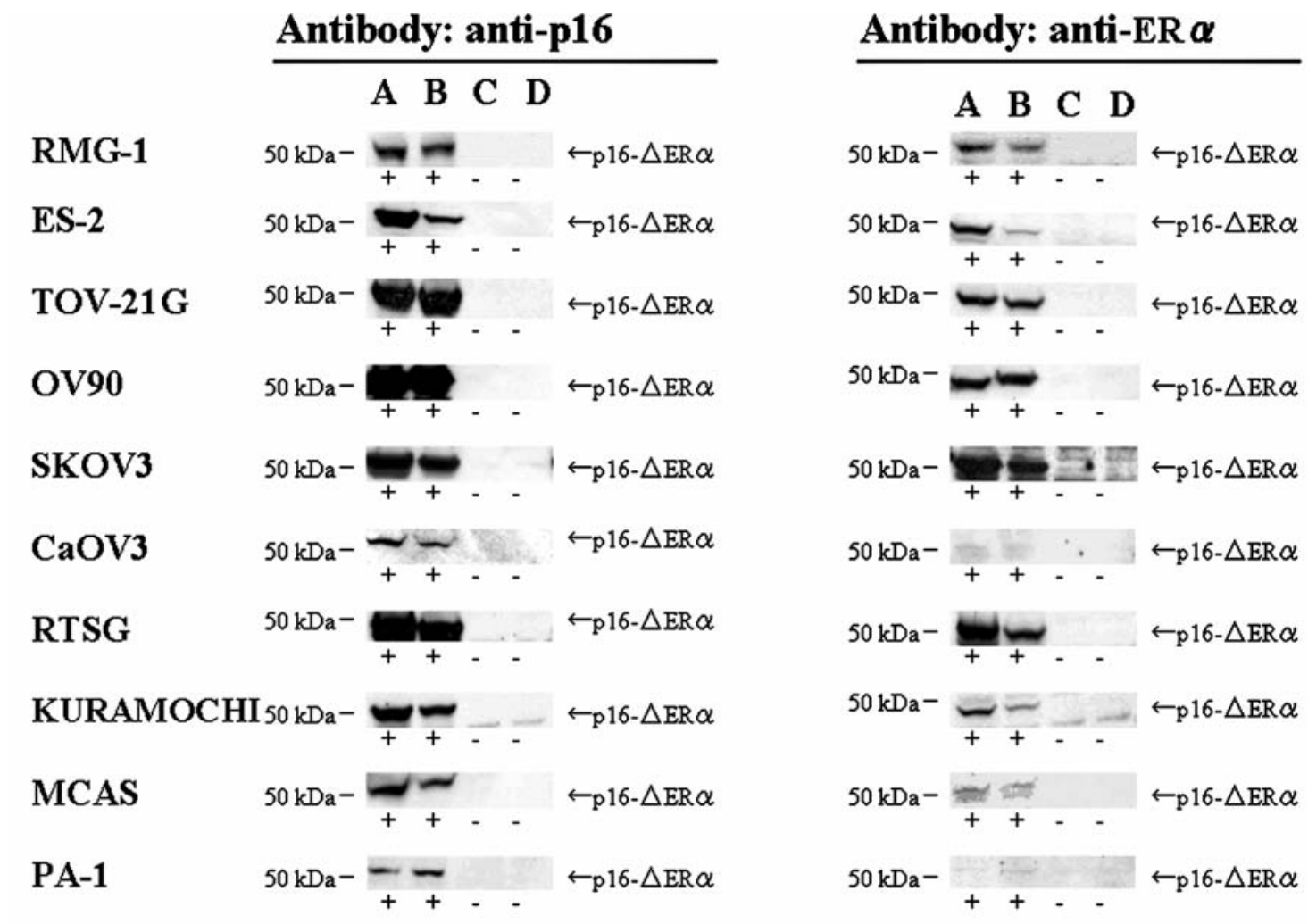

A; Infection(+) Estradiol(+) B; I(+)E2(-) C; I(-)E2(+) D; I(-)E2(-)Negative control

Figure 3. Western blot analysis using antibodies against p16INK4A and ER $\alpha$. The p16INK4A- $\Delta E R \alpha$ fusion protein was detected at the same position on both Western blots in all cell lines infected with the adenovirus (groups A and B). No bands were detected without infection (groups C and D).

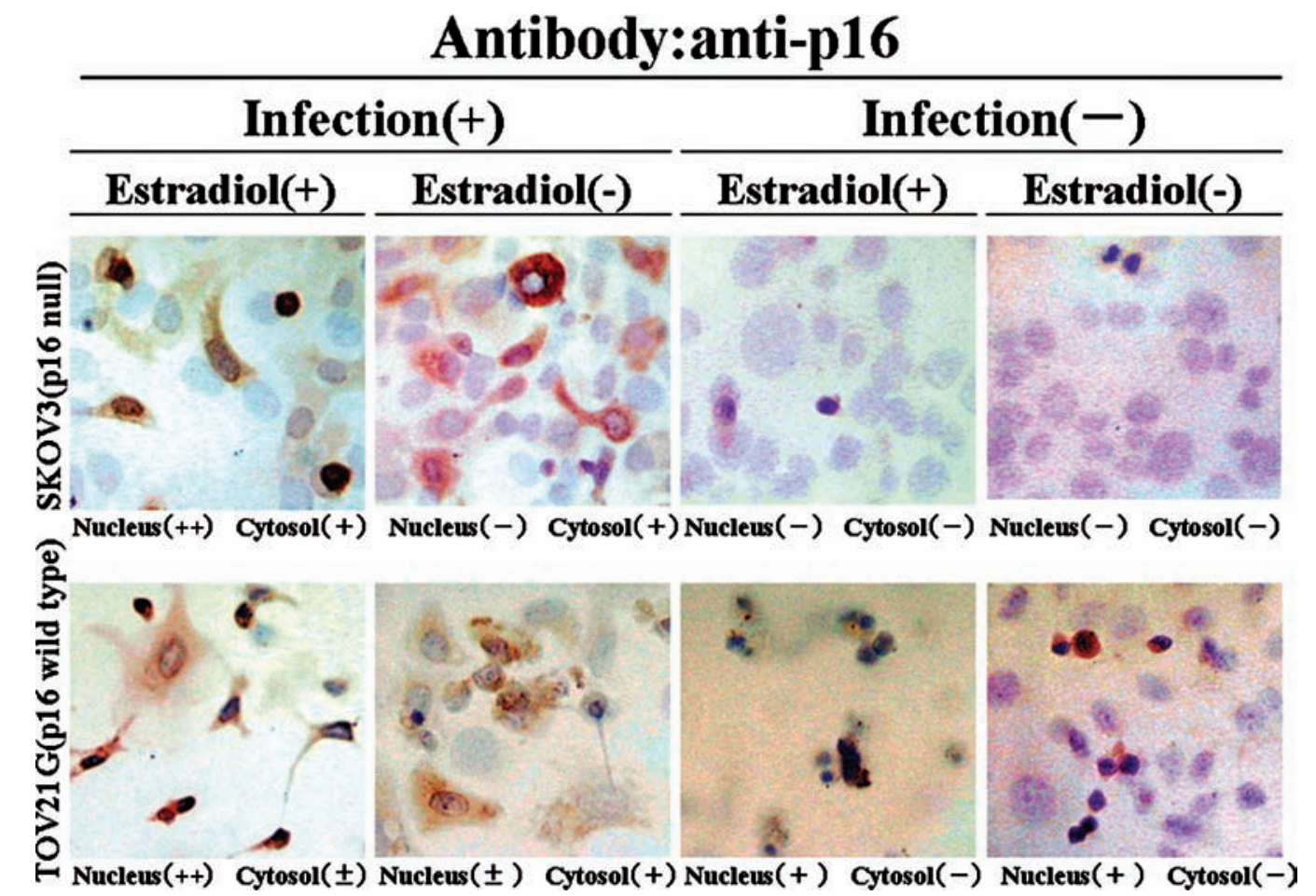

Figure 4. Immunohistochemical analyses of the intracellular localization of the fusion protein under estrogen-positive or -negative conditions. The cells were stained with an antibody against p16INK4A so that both the wild-type and the p16INK4A- $\Delta$ ER $\alpha$ fusion protein could be detected. In the four cell lines lacking wild-type p16INK4A (RMG-1, RTSG, SKOV3 and MCAS), positive staining was not detected in the absence of infection (groups C and D). After infection with the adenovirus and in the absence of 17ß-estradiol (group B), p16INK4A was detected in the cytosol of these cells, and when these infected cells were treated with 17B-estradiol (group A), p16INK4A was detected in the nucleus. This effect of 17B-estradiol was also observed in cell lines expressing wild-type p16INK4A (ES-2, TOV21G, OV90, CaOV3, KURAMOCHI and PA-1). 
A
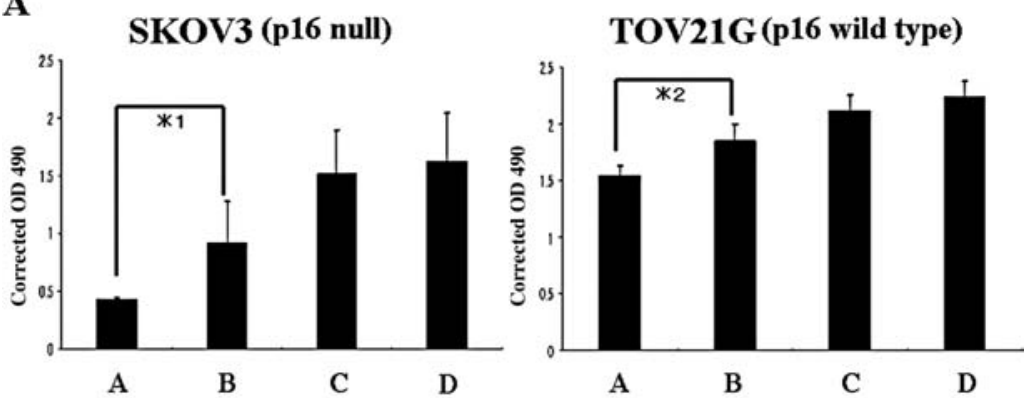

$* 1$ p $<0.001$

$* 2$. $<0.005$

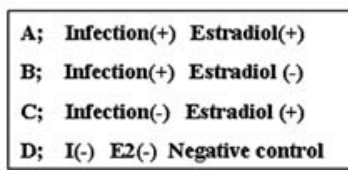

\section{B SKOV3(p16 null)}
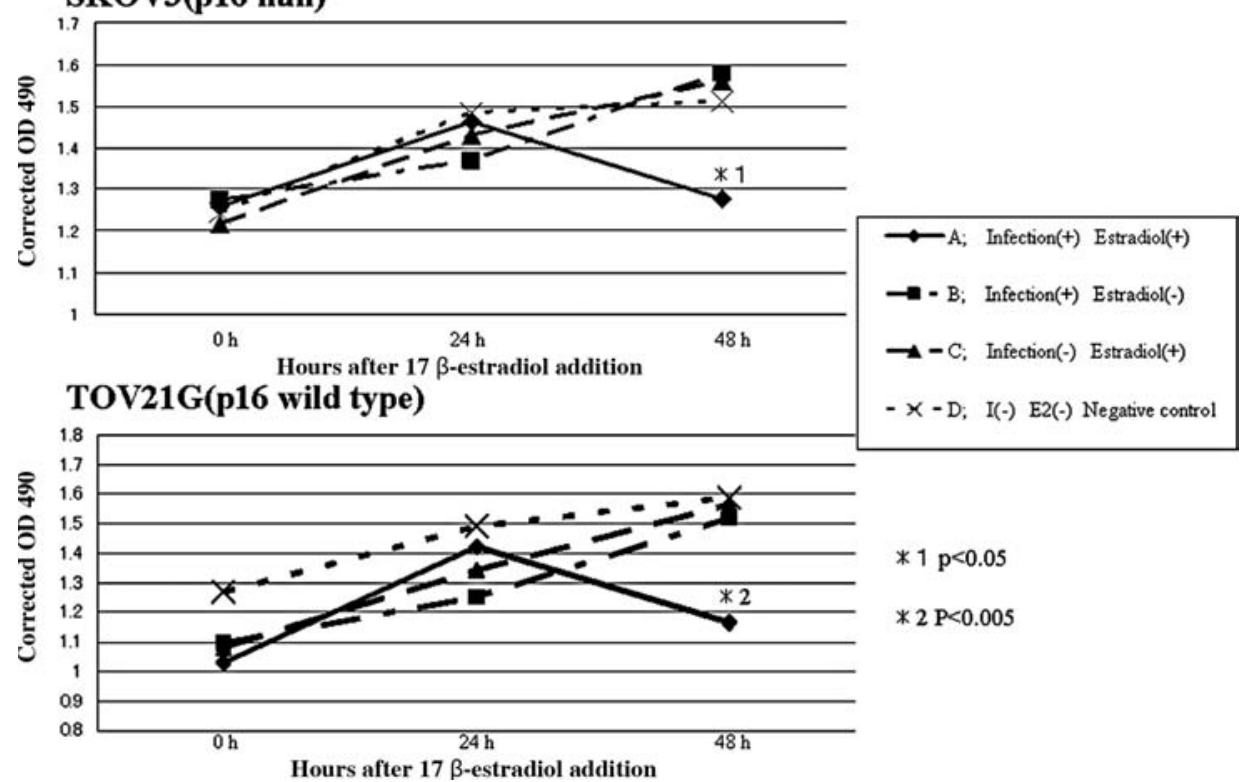

$* 1 \mathrm{p}<0.05$

* $2 \mathrm{P}<0.005$

Figure 5. Effect of the fusion protein on cell proliferation. Cell proliferation was examined in the four treatment groups using an MTS assay. (A) Forty-eight hours after the addition of $17 \mathrm{~B}$-estradiol ( $72 \mathrm{~h}$ after infection). (B) Time course of the effect at the indicated time points after infection and treatment with 173 estradiol. Forty-eight hours after the 173 -estradiol ( $72 \mathrm{~h}$ after infection), both of the average absorbance at $490 \mathrm{~nm}$ (MTS conversion) were significantly lower in group A (infected cells treated with 17ß-estradiol) than the other three groups. Results represent the averages of 10 replicates for each condition.

stimulus where p16INK4A can carry out its normal function of p16INK4A.

Growth suppression by the p16INK4A- $\Delta E R \alpha$ fusion protein. We next examined the proliferation of cells by an MTS assay. The proliferation of the four different groups of cells was assessed at various times after infection and treatment with $17 ß$-estradiol. When measured $48 \mathrm{~h}$ after the addition of $17 \mathrm{~B}$-estradiol (72 $\mathrm{h}$ after infection), the average of the absorbance at $490 \mathrm{~nm}$ (mitochondrial conversion of MTS) was significantly lower in group A than in the other three groups in SKOV3 and TOV21G cells (Fig. 5A and B). A similar tendency was observed in the other eight cell lines, but the differences were not statistically significant (data not shown). This suggests that $17 ß$-estradiol significantly suppresses growth in infected SKOV3 and TOV21G cells.

Western blot analysis of the level of phosphorylated $R b$ protein. When estrogen binds to p16INK4A- $\Delta \mathrm{ER} \alpha$ fusion proteins in the cytoplasm, they are translocated to the nucleus where they suppress cell growth. This suppression of cell growth is mediated by a decrease in the level of phosphorylated $\mathrm{Rb}$ due to inhibition of cyclin $\mathrm{D}-\mathrm{CDK} 4$ complexes (15) and blockage of the release of the transcription factor E2F (16). Therefore, we performed Western blotting with an antibody against phospho-Rb (Ser780), the site phosphorylated by cyclin D-CDK4 complexes (17), to determine whether the level of phosphorylated $\mathrm{Rb}$ protein is decreased in SKOV3 and TOV21G cells. As shown in Fig. 6, the level of phosphorylated $\mathrm{Rb}$ was remarkably lower in group $\mathrm{A}$ (infected and treated with 17ß-estradiol) than in the other three groups. These results suggested that the overexpression of the p16INK4A fusion protein in the nucleus suppresses cell growth by inhibiting the phosphorylation of Rb by cyclin D-CDK4 complexes.

Cell cycle effects of p16INK4A- $\Delta E R \alpha$ fusion proteins. The overexpression of p16INK4A protein leads to an arrest in the 


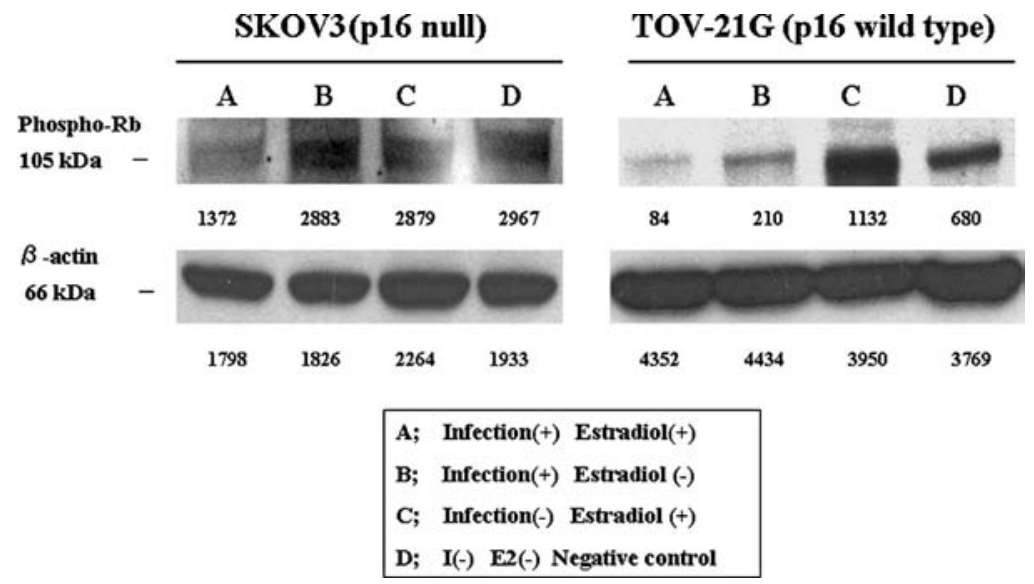

Figure 6. Phosphorylation of Rb in TOV21G and SKOV3 cells. Effect of expression of the fusion protein on phosphorylation of Rb. Rb phosphorylation in SKOV3 and TOV21G cells was examined by Western blotting using antibody against phospho-Rb (Ser780). Rb phosphorylation was substantially decreased in group A compared with groups B-D. The numbers below the bands represent the relative band intensities as determined by densitometry.

Flow cytometry

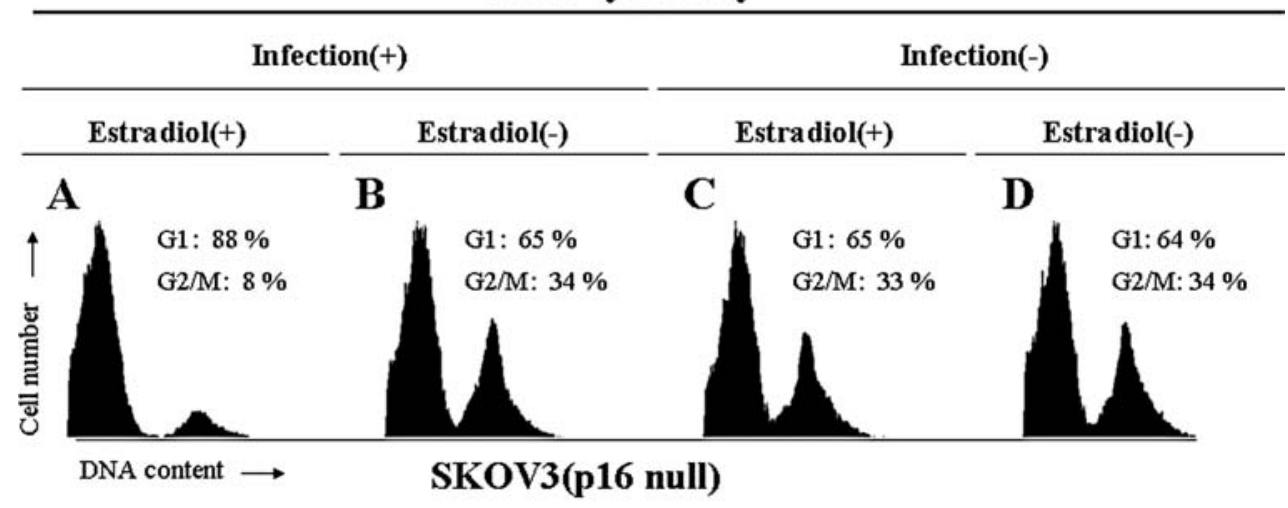

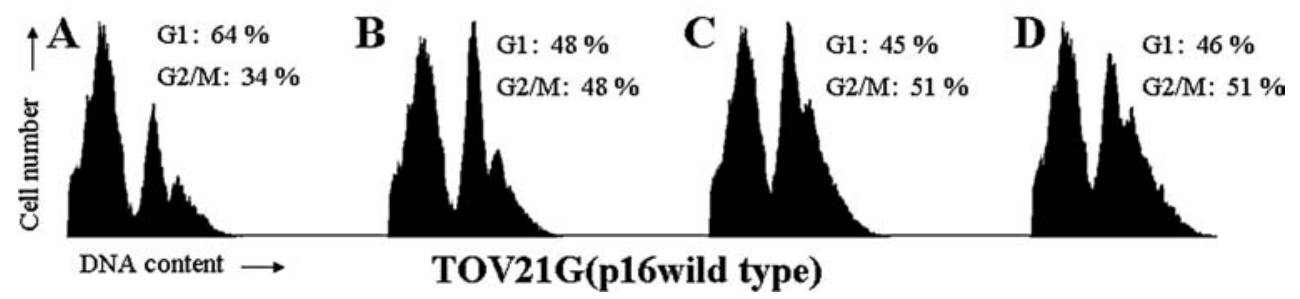

Figure 7. Effect of the fusion protein on cell cycle. (A) SKOV3, $48 \mathrm{~h}$ after the addition of 17ß-estradiol ( $72 \mathrm{~h}$ after infection), $88 \%$ of SKOV3 cells in group A were in G1 phase, compared with 64-65\% in groups B-D. Then, $8 \%$ of SKOV3 cells in group A were in G2/M phase, compared with 33-34\% in groups B-D. (B) TOV21G, $48 \mathrm{~h}$ after the addition of 17ß-estradiol (72 $\mathrm{h}$ after infection), $64 \%$ of TOV21G cells in group A were in G1 phase, compared with $45-48 \%$ in groups B-D. Then, $34 \%$ of TOV21G cells in group A were in G2/M phase, compared with 48-51\% in groups B-D.

G1 phase of the cell cycle (18). To investigate whether the $\mathrm{p} 16 \mathrm{INK} 4 \mathrm{~A}-\Delta \mathrm{ER} \alpha$ fusion protein induces $\mathrm{G} 1$ arrest at the presence of estrogen, we performed flow cytometric cell cycle analysis in SKOV3 and TOV21G cells. Forty-eight hours after the addition of 17ß-estradiol (72 $\mathrm{h}$ after infection), cells in groups A-D were harvested and the DNA content was determined. As we predicted, the p16INK4A fusion protein at the presence of estrogen (group A; infected and treated with 173 -estradiol) increased the fraction of cells in $\mathrm{G} 1$, and remarkably decreased the cell-population in G2/M. On the other hands, the absence of estrogen (group B; infected and without $17 ß$-estradiol) has no effects on cell-populations compared with negative control (group D) in either SKOV3 or TOV21G cells (Fig. 7). These results suggest that the overexpression of the p16INK4A fusion protein only in the nucleus induces cell cycle arrest.

\section{Discussion}

In the current studies, we established a new regulated functional gene expression system for gene therapy applications. This system employs p16INK4A fused at its C-terminus to the ligand-binding domain of the $\mathrm{ER} \alpha$ and is introduced into mammalian cells via a single adenoviral vector. We were able to establish cell lines expressing this fusion protein wherein the function of p16INK4A, a G1 cyclin-dependent 
kinase inhibitor, depended on the nuclear transport of the fusion protein in the presence of estrogen. Thus, these cell lines carry inducible expression of a protein that is a potential tumor suppressor. This system may be applied in the future to clinical trials of gene therapy in humans.

Gossen and Bujard (2) have demonstrated tight control of gene expression in mammalian cells by using an inducible system based on the Tn10-specified tetracycline resistance operon of Escherichia coli. In this system, gene expression is regulated by a hybrid transcription factor, tTA, which consists of the transactivation domain of herpes simplex virus VP16 $(2,19,20)$ fused to the carboxy terminus of the tetracycline repressor, tetR. To use this system, cell lines that stably express tTA must first be established. Furthermore, efficient expression of tTA is toxic to cells, probably because of the squelching effect of the VP16 transactivation domain in tTA $(2,19,21)$. Once cell lines that stably express tTA have been established, the gene of interest under control of the tetOcontaining promoter must be introduced.

To eliminate some of these problems, we constructed a hybrid protein, p16INK4A- $\Delta \mathrm{ER} \alpha$, containing the ligandbinding domain of the ER $\alpha$ fused to the carboxy terminus of p16INK4A. When the ligand-binding domains of steroid hormone receptor family members are fused with heterologous proteins, their expression can regulated by the cognate hormone (22). It has been hypothesized that the disruption of the normal functions of the wild-type protein are due to the association of the hormone receptor ligand-binding domain with a complex of cellular proteins including heat shock protein 90 in the absence of the cognate hormone (22). Hormone-binding results in the release of the cellular protein complex from the fusion protein, leading to restoration of the protein's normal functions. By placing the ER ligand-binding domain adjacent to the p16INK4A, it seemed likely that we could place the function of p16INK4A under hormonal control.

Using this system, we established ovarian cancer cell lines stably expressing p16INK4A, which has been shown to cause the arrest of cancer cells (23). A major consideration for this system was whether the conformation of the p16INK4A protein would be affected by fusion with the $\Delta \mathrm{ER} \alpha$ protein. We found that the $\mathrm{p} 16 \mathrm{INK} 4 \mathrm{~A}-\Delta \mathrm{ER} \alpha$ fusion protein is expressed in the cytosol after infection with the adenovirus vector and that it is quickly translocated into the nucleus in the presence of estrogen. Under these conditions, p16INK4A plays an essential role in the cell, causing the infected cancer cells to undergo cell cycle arrest.

\section{Acknowledgments}

We thank Dr Pierre Chambon for providing plasmid hER/ pSG5. This work was supported by grants-in-aid for scientific research (nos. 13671694 and 15591730) from the Ministry of Education, Culture, Sports, Science and Technology of Japan.
2. Gossen $\mathrm{M}$ and Bujard $\mathrm{H}$ : Tight control of gene expression in mammalian cells by tetracycline-responsive promoters. Proc Natl Acad Sci USA 89: 5547-5551, 1992.

3. Wang Y, O'Malley BW Jr, Tsai Y and O'Malley BE: A regulatory system for use in gene transfer. Proc Natl Acad Sci USA 91: 8180-8184, 1994.

4. Burcin MM, Schiedner G, Kochanek S, Tsai SY and O'Malley BW: Adenovirus-mediated regulatable target gene expression in vivo. Proc Natl Acad Sci USA 96: 355-360, 1999.

5. Iida A, Chen ST, Friedmann T and Yee JK: Inducible gene expression by retrovirus-mediated transfer of a modified tetracycline-regulated system. J Virol 70: 6054-6059, 1996.

6. Hama S, Heike Y, Naruse I, Takahashi M, Yoshioka H and Arita K: Adenovirus mediated p16 gene transfer prevents druginduced cell death trough G1 arrest in human glioma cells. Int J Cancer 77: 47-54, 1998.

7. Harada H, Nakagawa K, Iwata S, Saito M, Kumon Y and Sakai S: Restoration of wild-type p16 down-regulate vascular endothelial growth factor expression and inhibits angiogenesis in human glioma. Cancer Res 59: 3783-3789, 1999.

8. Kobayashi S, Shirasawa H, Sashiyama H, Kawashima H, Kaneko K and Asano T: p16INK4a expression adenovirus vector to suppress pancreatic cancer cell proliferation. Clin Cancer Res 5: 4182-4185, 1999.

9. Modesitt SC, Ramirz P, Zu Z, Bodurka-Bevers D, Gershenson D and Wolf JK: In vitro and in vivo adenovirus-mediated p53 and p16 tumor suppressor therapy in ovarian cancer. Clin Cancer Res 7: 1765-1772, 2001.

10. Krishan A: Rapid flow cytofluorometric analysis of mammalian cell cycle by propidium iodide staining. J Cell Biol 66: 188-193, 1975.

11. Kanuma K, Nishida J, Mizunuma H, Ibuki Y and Wake N: Role of the $\mathrm{Rb}$-mediated cell cycle regulatory pathway in human ovarian cancer cell lines. Ann Cancer Res Ther 6: 51-57, 1997.

12. Kanuma T, Nishida J, Gima T, Barrett JC and Wake N: Alterations of the p16INK4A gene in human ovarian cancers. Mol Carcinog 18: 134-141, 1997.

13. Kumar V, Green S, Staub A and Chambon P: Localization of the oestradiol-binding and putative DNA-binding domains of the human oestrogen receptor. EMBO J 5: 2231-2236, 1986.

14. Kumar V, Green S, Stack G, Berry M, Jin J and Chambon P: Functional domains of the human estrogen receptor. Cell 51: 941-951, 1987.

15. Serrano M, Lin AW, McCurrach ME, Beach D and Lowe SW: Oncogenic ras provokes premature cell senescence associated with accumulation of p53 and p16INK4a. Cell 88: 593-602, 1997.

16. Dyson N: The regulation of E2F by pRB-family proteins. Genes Dev 12: 2245-2262, 1998.

17. Kitagawa M, Higashi H, Jung H, Suzuki-Takahashi I, Ikeda M and Tamai K: The consensus motif for phosphorylation by cyclin D1-Cdk4 is different from that for phosphorylation by cyclin A/E-Cdk2. EMBO J 15: 7060-7069, 1996.

18. Medema RH, Herrera RE, Lam F and Weinberg RA: Growth suppression by p16ink4 requires functional retinoblastoma protein. Proc Natl Acad Sci USA 92: 6289-6293, 1995.

19. Gill $\mathrm{G}$ and Ptashne M: Negative effect of the transcriptional activator GAL4. Nature 334: 721-724, 1988.

20. Triezenberg SJ, Kingsbury RC and McKnight SL: Functional dissection of VP16, the transactivator of herpes simplex virus immediate early gene expression. Genes Dev 2: 718-729, 1988.

21. Shockett P, Difilippantonio M, Hellman N and Schatiz DG: A modified tetracycline-regulated system provides autoregulatory, inducible gene expression in cultured cells and transgenic mice. Proc Natl Acad Sci USA 92: 6522-6526, 1995.

22. Mattioni T, Louvion J and Picard D: Regulation of protein activities by fusion to steroid binding domains. Methods Cell Biol 43: 335-352, 1994.

23. Wolf JK, Kim TE, Fightmaster D, Bodurka D and Gershenson DM: Growth suppression of human ovarian cancer cell lines by the introduction of p16 gene via a recombinant adenovirus. Gynecol Oncol 73: 27-34, 1999.

\section{References}

1. Goverdhara S, Puntel M, Xiong W, et al: Regulatable gene expression systems for gene therapy applications: Progress and future challenges. Mol Ther 12: 189-211, 2005. 